\title{
The Effect of Voluntary and Forced Exercise on the Expression Level of NCAM-PSA Protein in the Neuromuscular Junction of Soleus Muscle in a Mice Experimental Autoimmune Encephalomyelitis Model
}

Farzaneh Torabimehr', Mohammad Reza Kordi ${ }^{*}$, Reza Nouri², Jafar $\mathbf{A i}^{3}$, Behnam Bakhtiari Moghadam ${ }^{4}$, Sadegh Shirian $^{4 \cdot 5: 6}$

${ }^{1}$ Department of Neuromuscular Exercise Physiology, Kish International Campus, Tehran University, Kish Island, Iran ${ }^{2}$ Department of Exercise Physiology, University of Tehran, Tehran, Iran

${ }^{3}$ Department of Tissue Engineering and Applied Cell Sciences, School of Advanced Technologies in Medicine, Tehran University of Medical Sciences, Tehran, Iran

${ }^{4}$ Department of Pathology, School of Veterinary Medicine, Shahrekord University, Shahrekord, Iran

${ }^{5}$ Shiraz Molecular Pathology Research Center, Dr Daneshbod Pathol Lab, Shiraz, Iran

${ }^{6}$ Shefa Neuroscience Research Center, Khatam Alanbia Hospital, Tehran, Iran

\section{Article Info:}

\section{A BSTRACT}

Introduction: It has been suggested the neural cell adhesion molecules (NCAM) are one of the main factors that play a role in progress or control of neurodegenerative diseases, such as multiple sclerosis (MS). It has been recently shown that exercises increase the NCAM expression in myofibres. This study aimed to investigate the effect of forced and voluntary training on the accumulation of NCAM-PSA in the neuromuscular junction in soleus muscles in pre and post-induction periods of an experimental autoimmune encephalomyelitis (EAE) mice model. Materials and Methods: A total of 40 female C57BL/6 mice were randomly divided into four groups including, induced control groups without EAE induction, induced EAE without training, induced EAE with forced, and voluntary training groups. Myelin oligodendrocyte glycoprotein peptide 35-55 (200 $\mu \mathrm{g}$ in saline) was injected subcutaneously at the base of the tail of each mouse. Forced and voluntary pieces of training were done using a swimming program and treadmill, respectively. The mice were euthanized and the soleus muscles were then isolated for investigation of protein expression using IHC. Results: In the control group, a significant increase in the mean percentage expression of NCAM-PSAin pre and post-soleus muscle was detected compared to the EAE with and without training as well as EAE without intervention group. The mean expression level of NCAM-PSA in the pre and post-soleus muscle were significantly increased in rat with treadmill group compared to the swimming group. The lowest expression was detected in EAE without intervention. Conclusion: The findings of the present study showed that voluntary training increases the expression levels of NCAM-PSA in the neuromuscular junction of the soleus muscle. Further studies are needed to investigate the effect of NCAM-PSA expression changes following exercise on muscular function in MS.

\section{Key words:}

1. Exercise

2. Muscles

3. Encephalomyelitis

*Corresponding Author: Mohammad Reza Kordi

E-mail:mrkordi@ut.ac.ir 
اثر ورزش اختيارى و اجبارى بر بيان سـطح يروتئين NCAM-PSA در اتصالات عصب به عضله

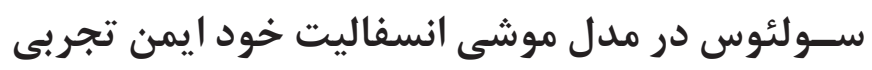

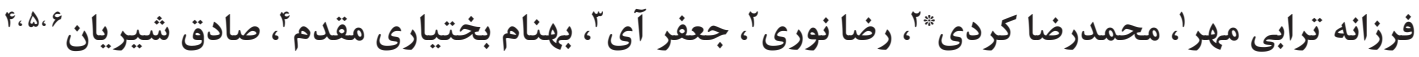

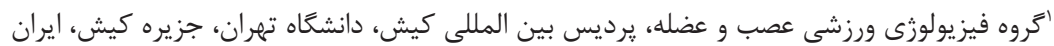

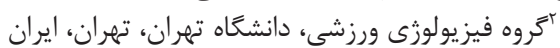

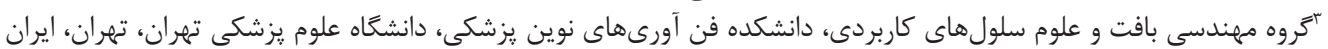

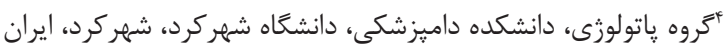

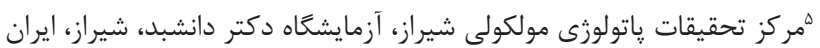

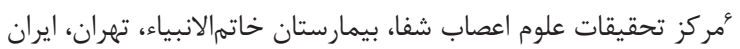

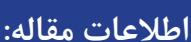

كليد وازهها:

1

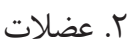

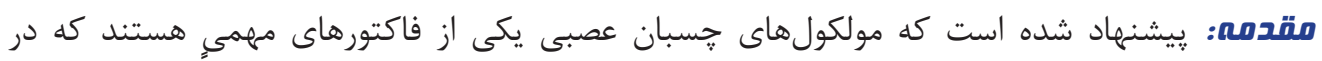

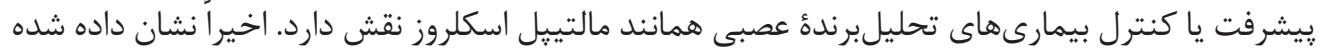

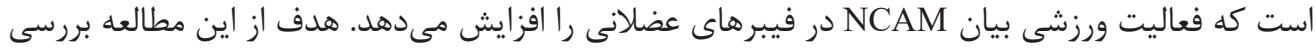

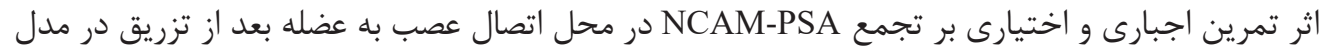

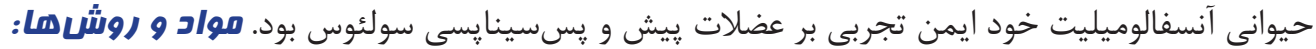

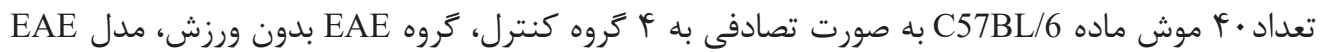

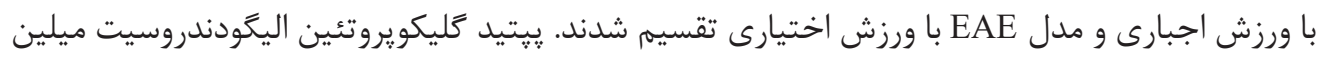

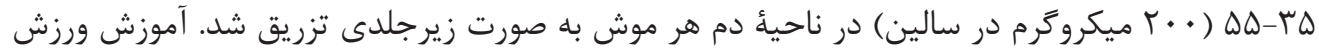

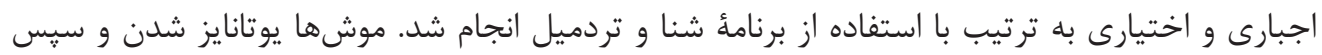

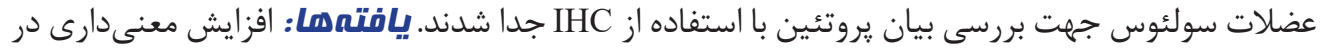

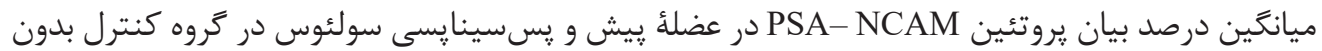

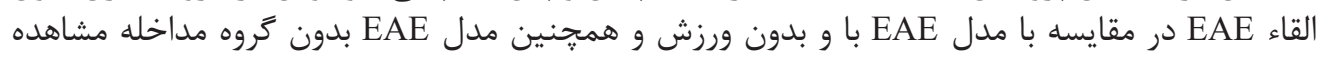

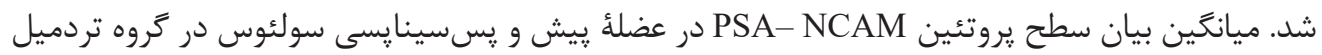

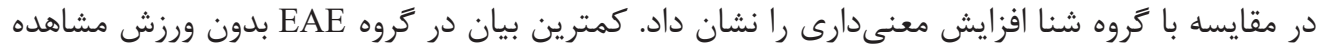

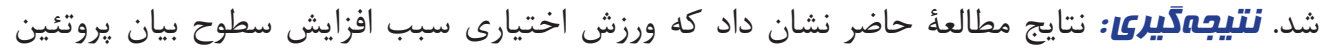
NCAM-PSA 


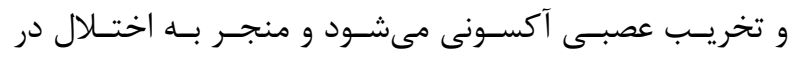

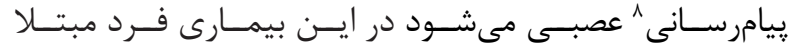

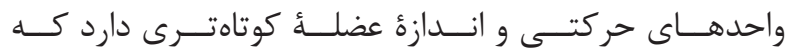

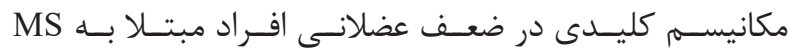

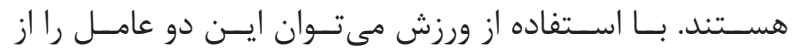

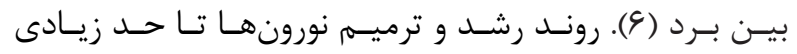

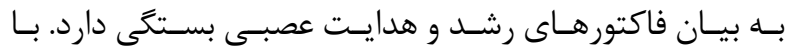

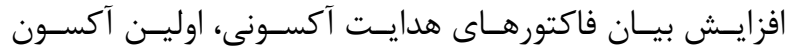

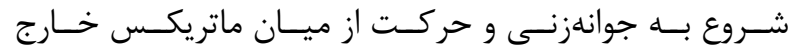

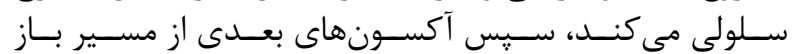

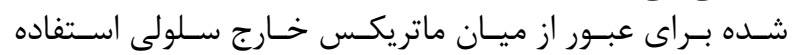

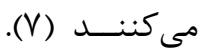

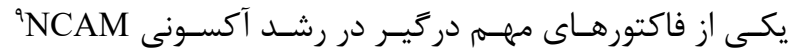

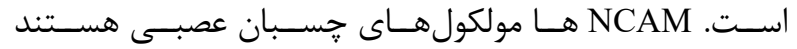

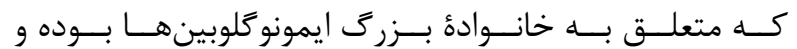

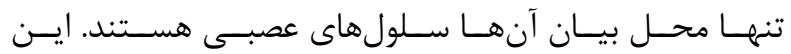

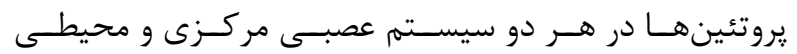

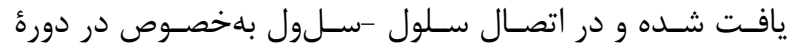

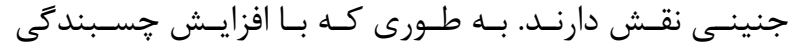

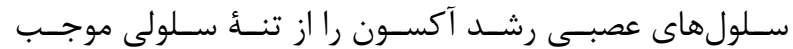

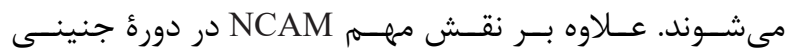

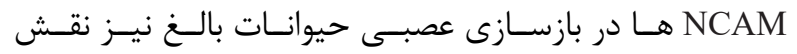

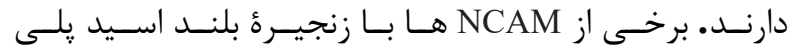

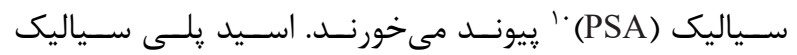

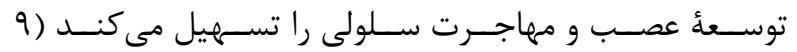

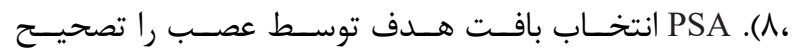

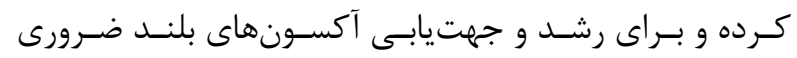

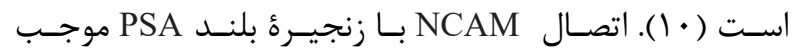

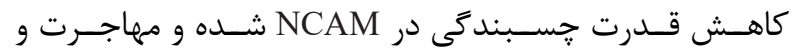

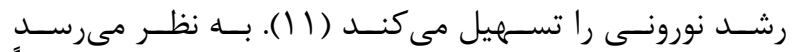

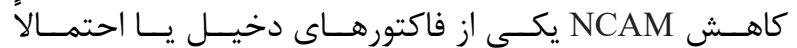

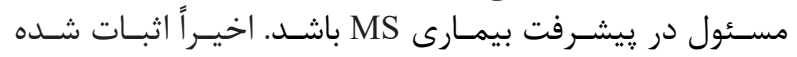

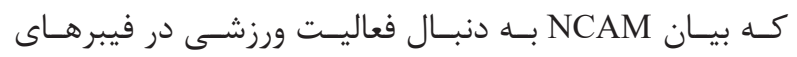
عضلانسى افزايـش مى ليابـــ (1).

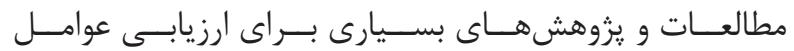

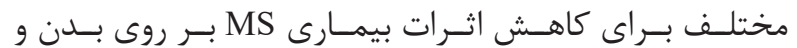

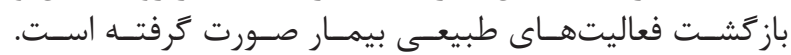

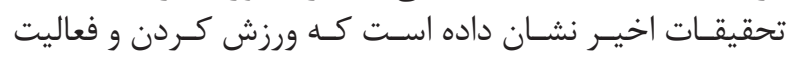

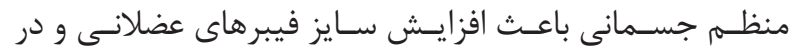

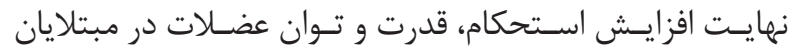

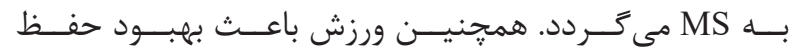

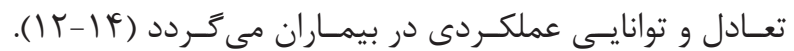

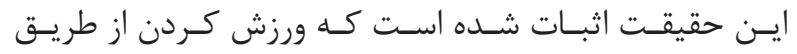

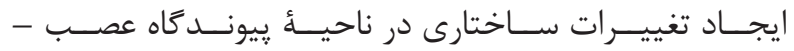

${ }^{1}$ Blood-brain barrier

${ }^{2}$ Multiple sclerosis

${ }^{3}$ Neuroantigen reactive

${ }^{4}$ Central nervous system

${ }^{5}$ Cerebrospinal fluid

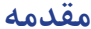

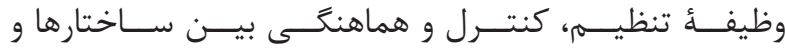

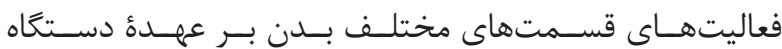

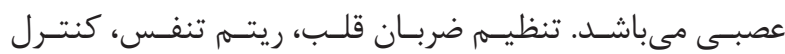

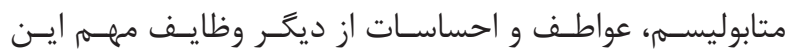

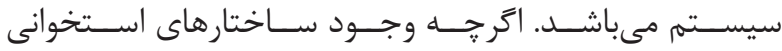

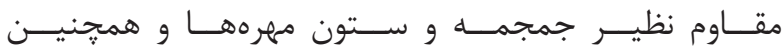

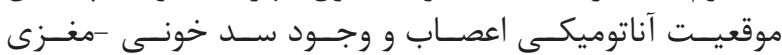

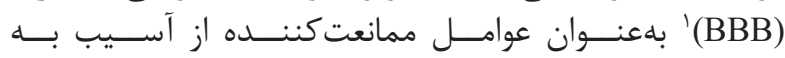

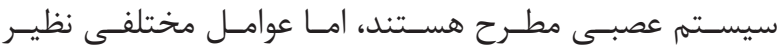

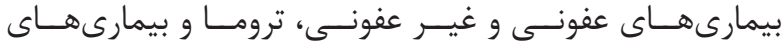

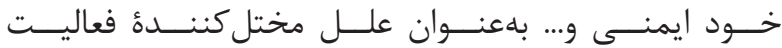

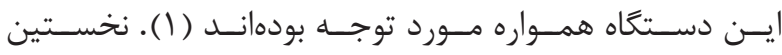

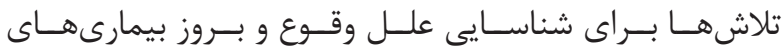

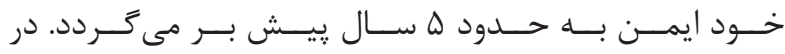

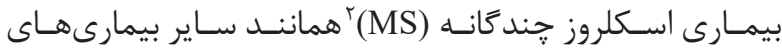

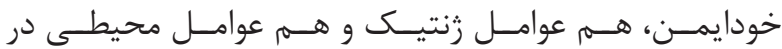

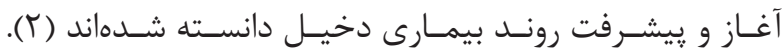

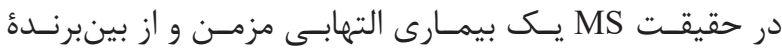

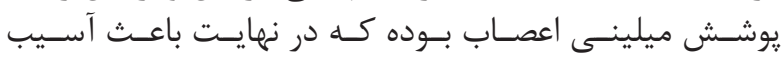

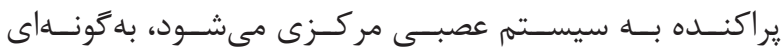

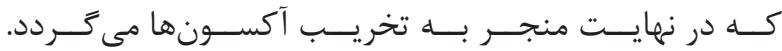

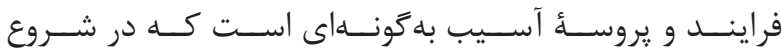

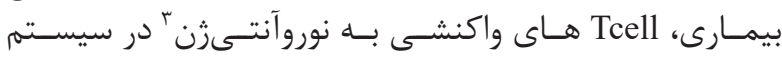

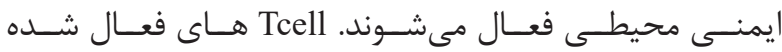

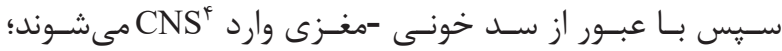

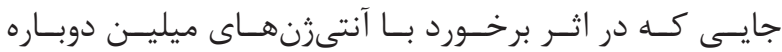

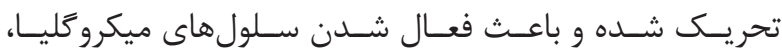

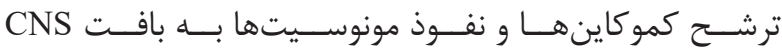

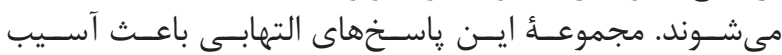

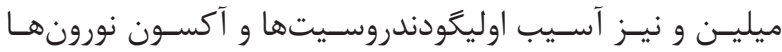

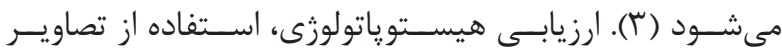
MRI

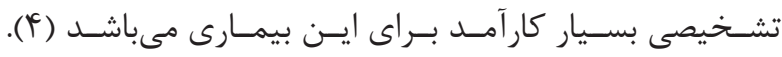

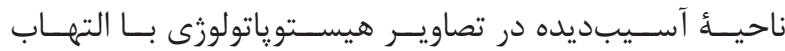

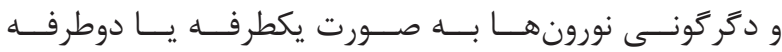

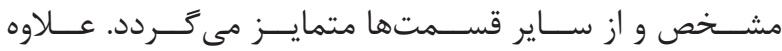

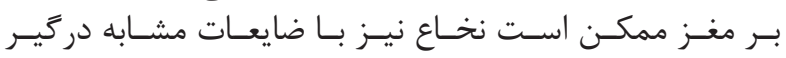

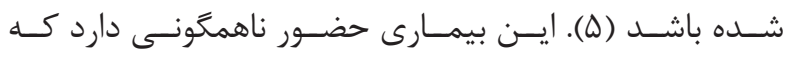

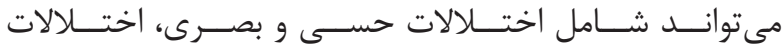

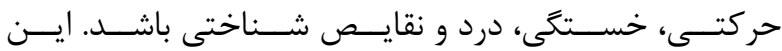

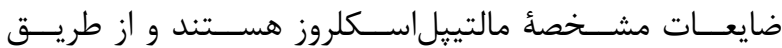

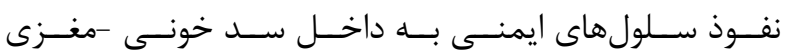

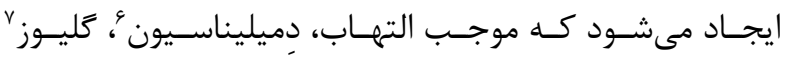

${ }^{6}$ Demyelination

${ }^{7}$ Gliosis

${ }^{8}$ Signaling

${ }^{9}$ Neural cell adhesion molecule

${ }^{10}$ Polyacial acid 


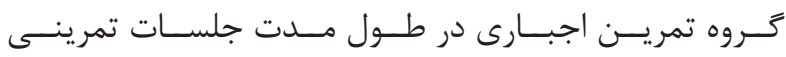

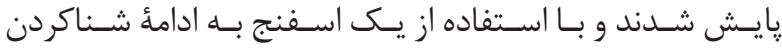

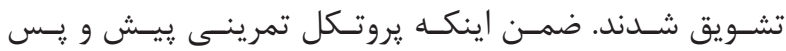

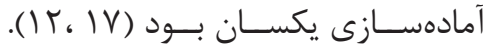

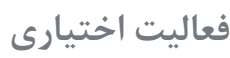

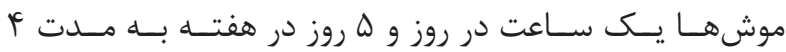

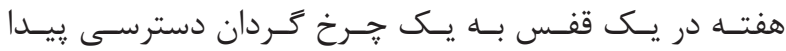

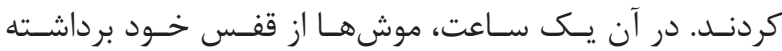

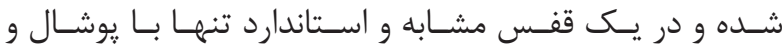

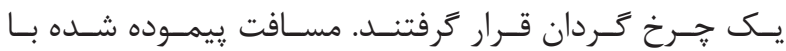

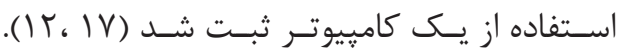

نحوة القاى EAE

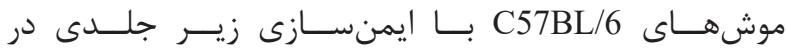

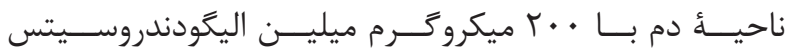

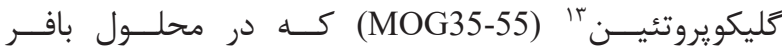

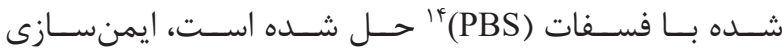

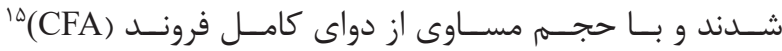

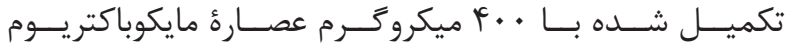

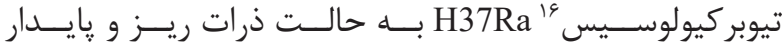

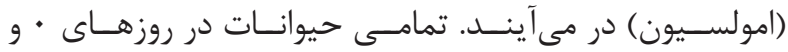

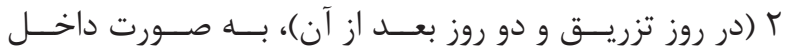

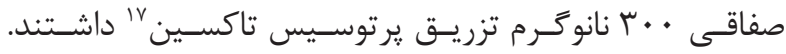

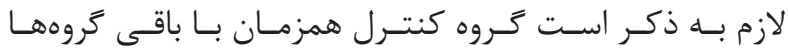

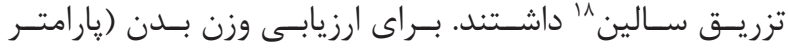

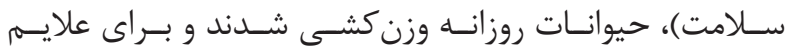

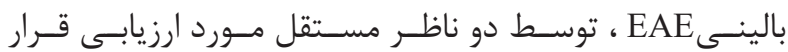

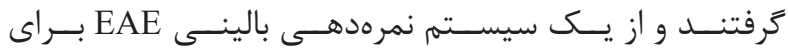

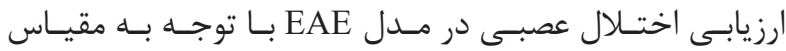

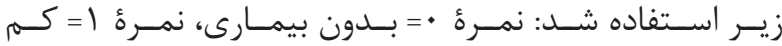

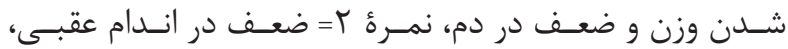

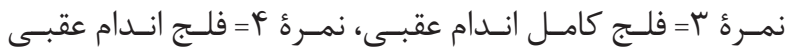

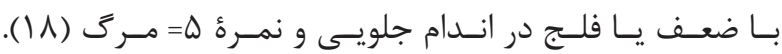
نمونهبردارى بافت بــراى انجـام رنتَآميـزى شـيميايى و مشـــص كـردن محــل

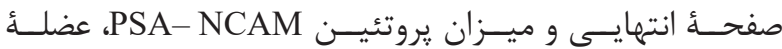

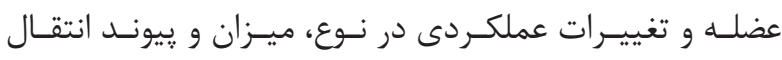

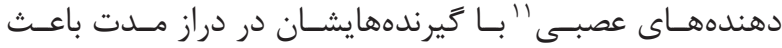

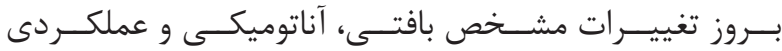

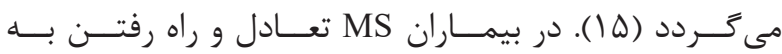

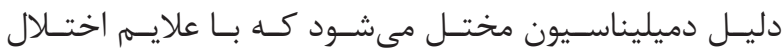

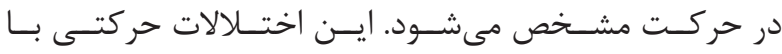

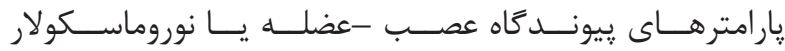

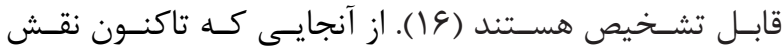

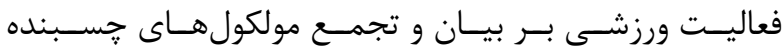

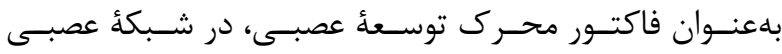

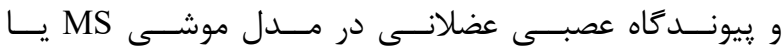
EAE ${ }^{r}$

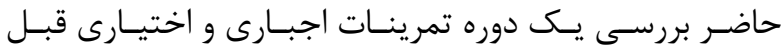

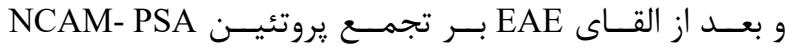

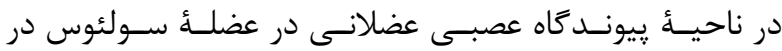

$$
\text { مــدل موشـى بـود. }
$$

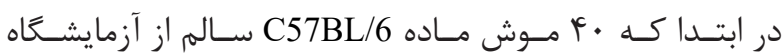

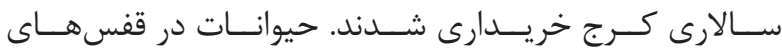

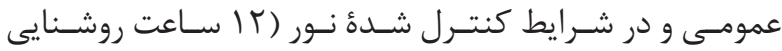

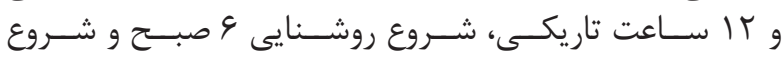

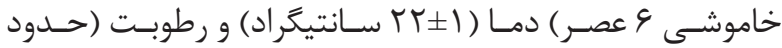

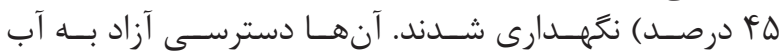

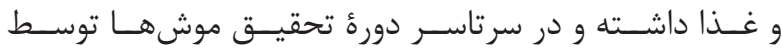

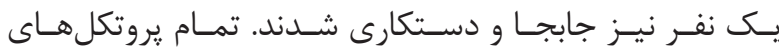

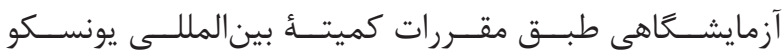

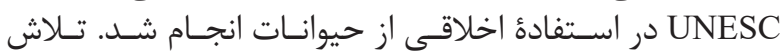

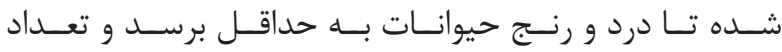

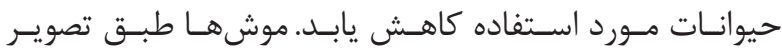

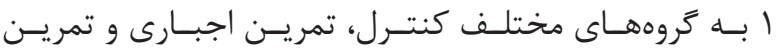

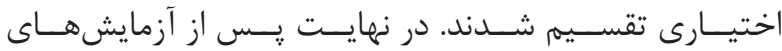

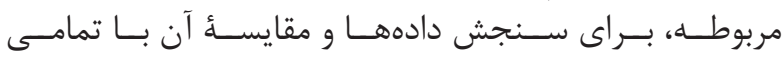

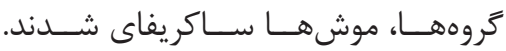

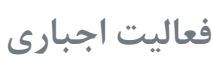

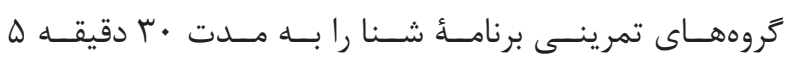

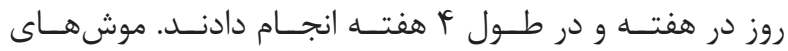

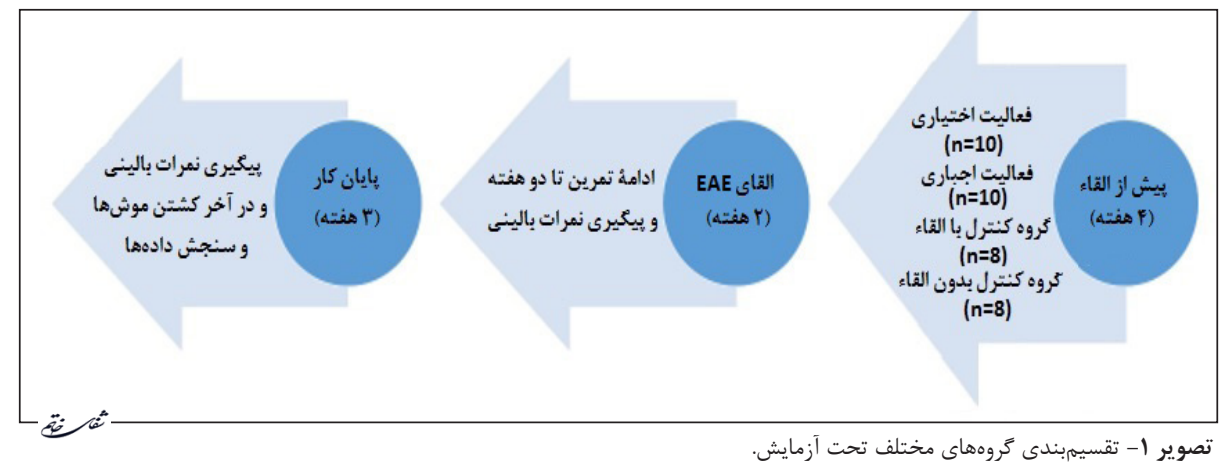

${ }^{11}$ Neurotransmitters

${ }^{12}$ Experimental autoimmune encephalomyelitis

${ }^{13}$ Myelin oligodendrocytes glycoprotein

${ }^{14}$ Phosphate-buffered saline
${ }^{15}$ Complete Freund's adjuvant

${ }^{16}$ Mycobacterium tuberculosis

${ }^{17}$ Pertussis toxin

${ }^{18}$ Saline 


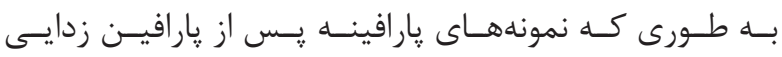

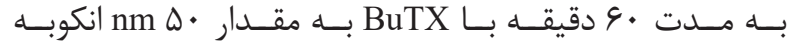

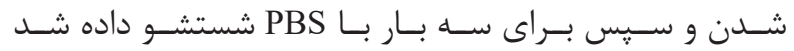

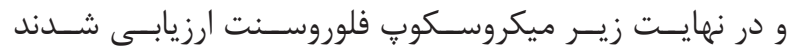

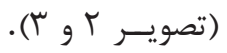

بيان NCAM-PSA

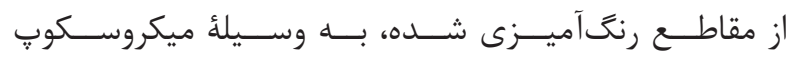

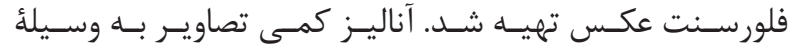

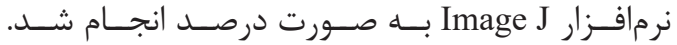
تجزيه و تحليل دادهها

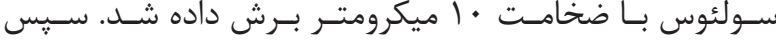

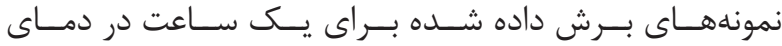

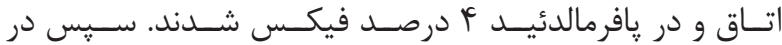

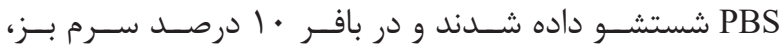
BSA

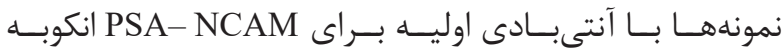

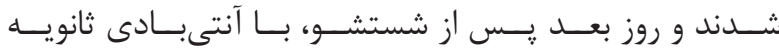

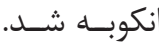

مشخص كردن صفحة انتهايى صفحــُ انتهايـى بـا اسـتفاده از آلفـا بونغاروتوكسـين (BuTX)

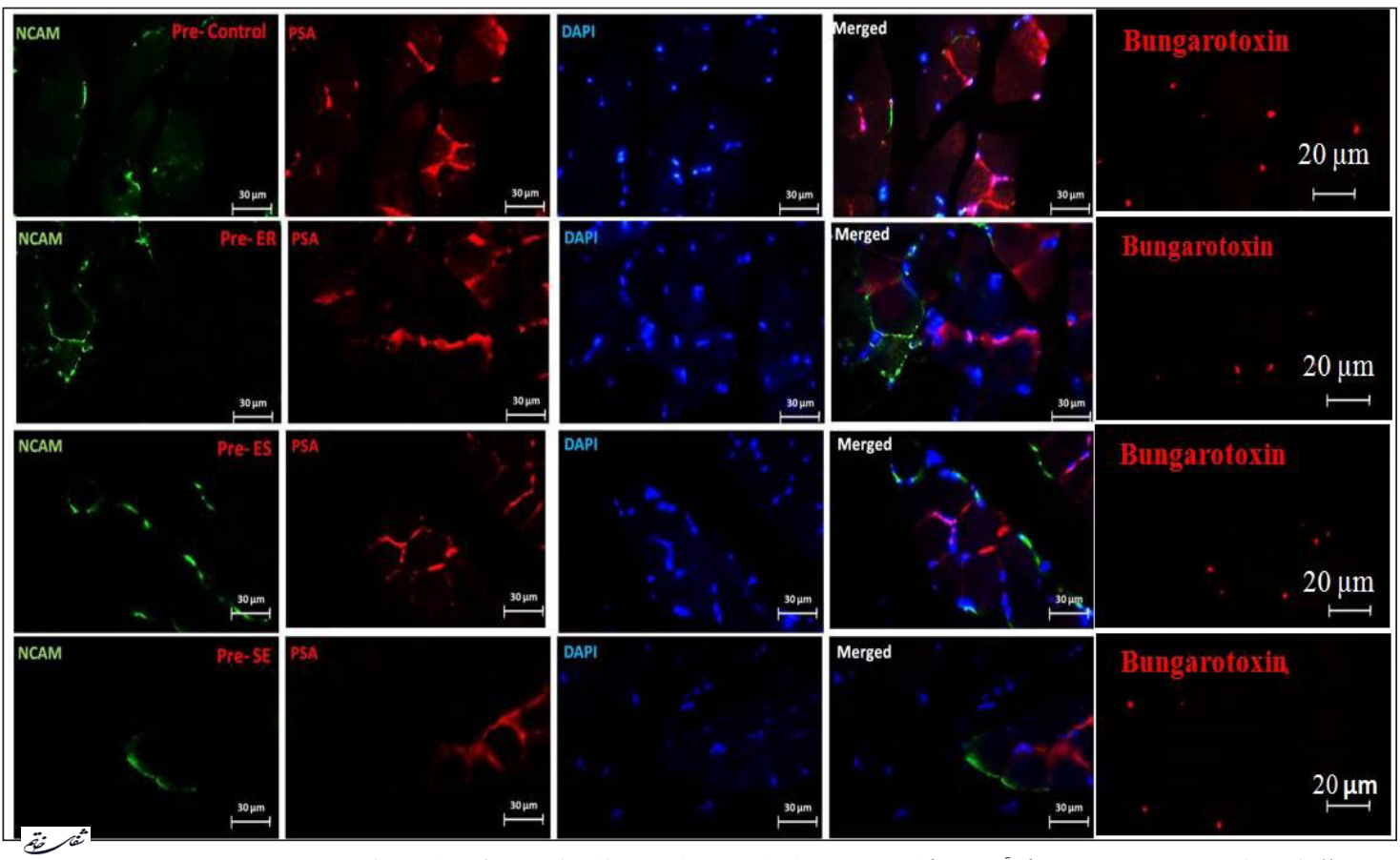

تصوير r- ايمونوراكتيويتى NCAM-PSA و رنگًآميزى بونغاروتوكسين در ناحية بيشسينايسى عضلة سولئوس در كروههاى مختلف.

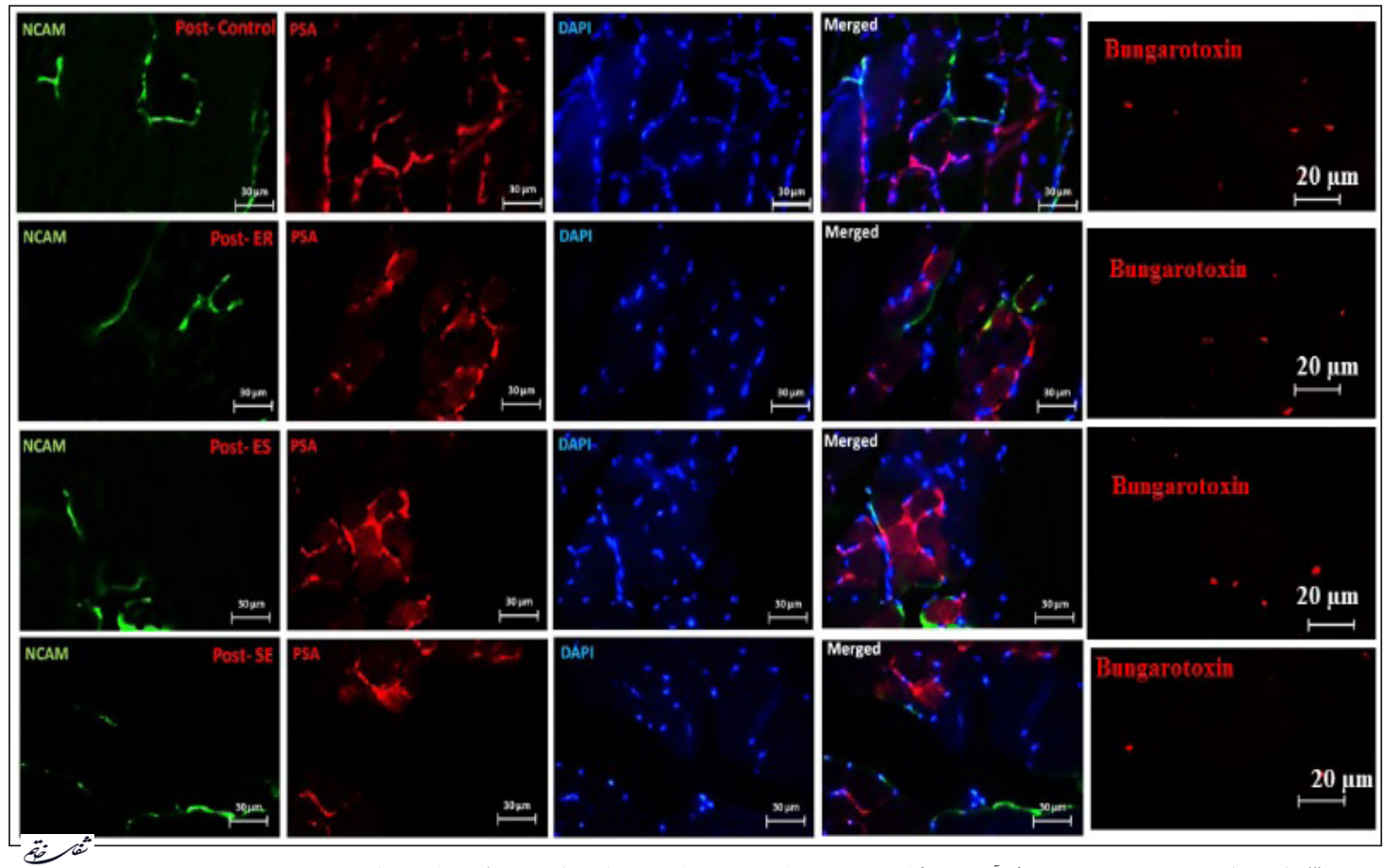

تصوير بـ ايمونوراكتيويتى NCAM-PSA و رنكَآميزى بونكاروتوكسين در ناحئ بِسسينايسى عضلة سولئوس در كروههاى مختلف. 


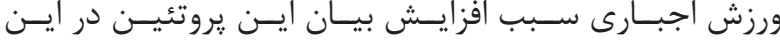

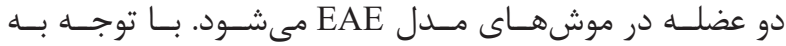

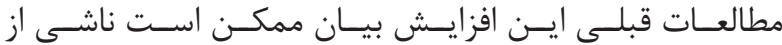

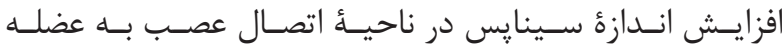

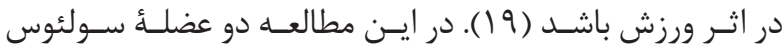

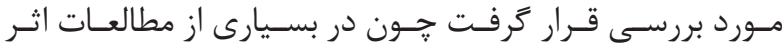

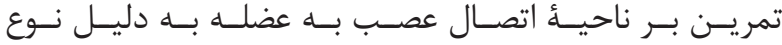

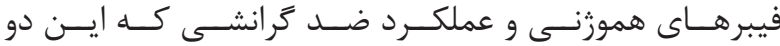

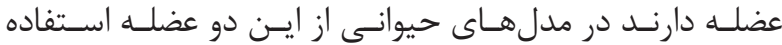

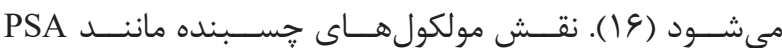

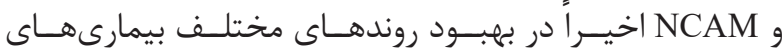

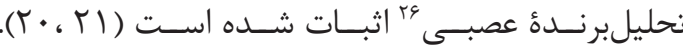

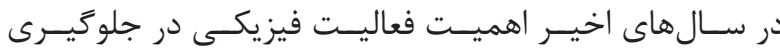

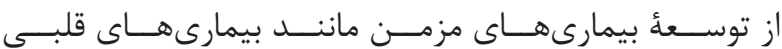

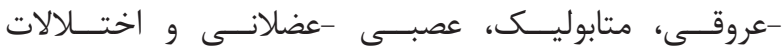

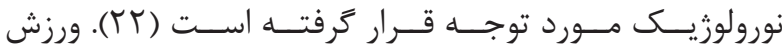

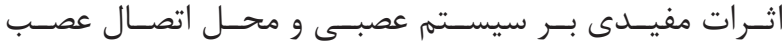

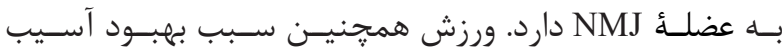

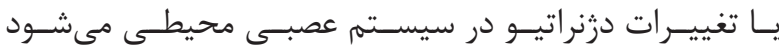

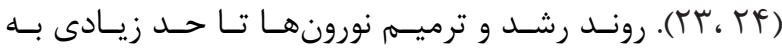

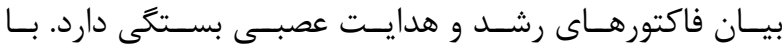

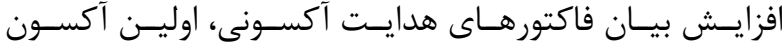

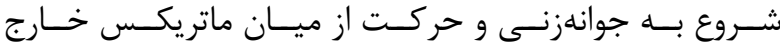

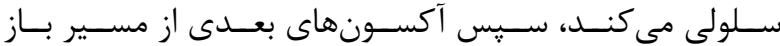

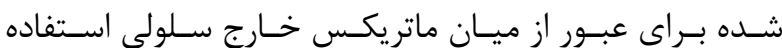

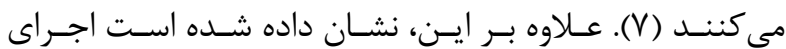

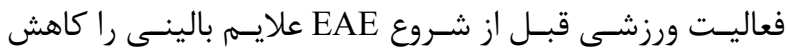

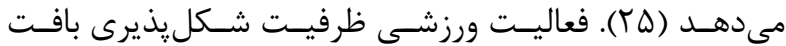

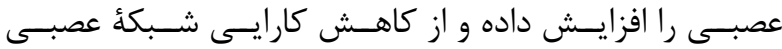

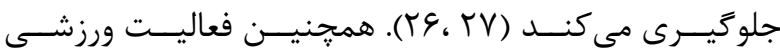

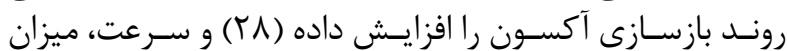

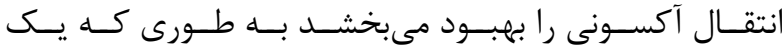

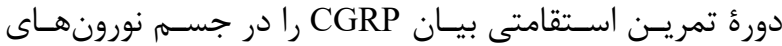

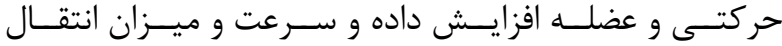

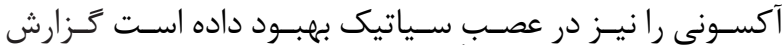

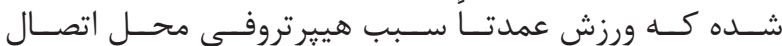

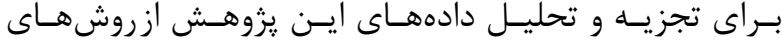

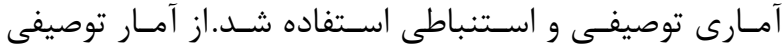

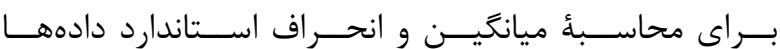

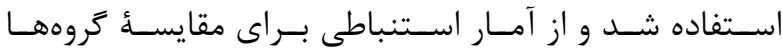

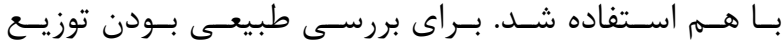

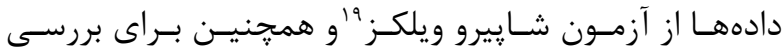

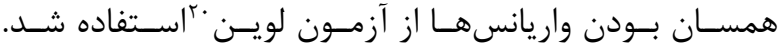

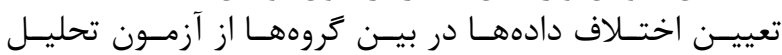

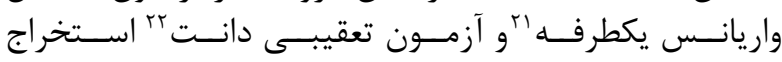

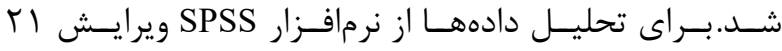

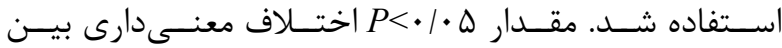

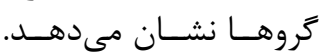

يافته ها

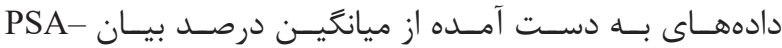
NCAM

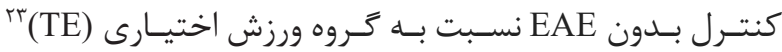

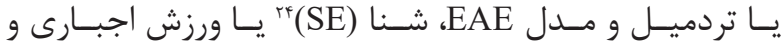

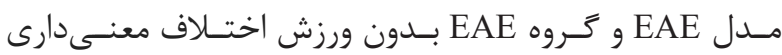

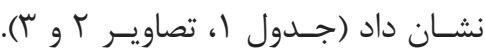
بحث و نتيجه كيرى

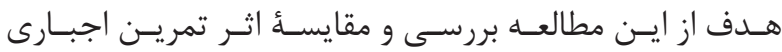

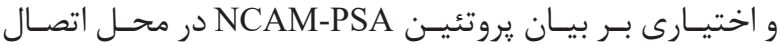

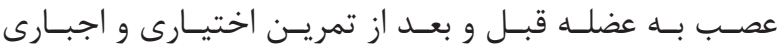

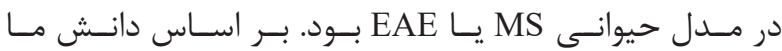

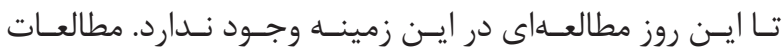

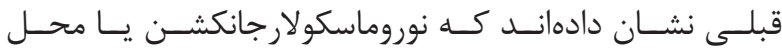

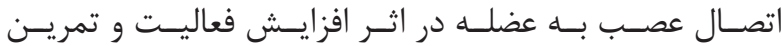

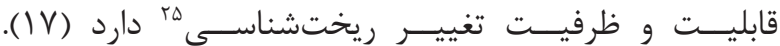

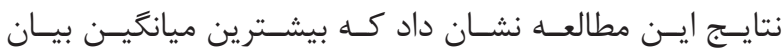

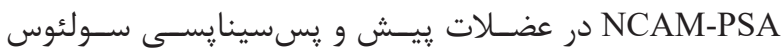

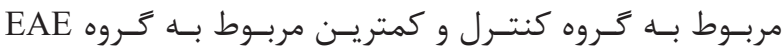

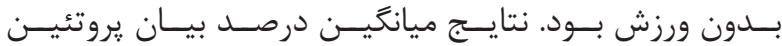

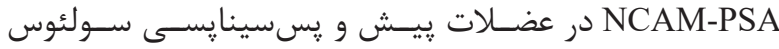

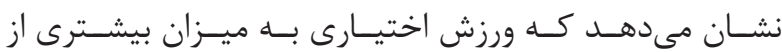

جدول ا- ميانكين درصد بيان NCAM-PSA در كروههاى مختلف حروف متفاوت نشاندهندة اختلاف معنى دار در هر رديف است (ه •/P>P).

\begin{tabular}{|c|c|c|c|}
\hline \multicolumn{4}{|c|}{ NCAM -PSA ييشىسينايسى سولئوس } \\
\hline كنترل بدون القاى EAE & تروه كنترل EAE بدون ورزش & كروه EAE و ورزش اختيارى & كروه EAE با ورزش اجبارى (شنا) \\
\hline $\mathrm{a} 91 / \mathrm{r} \pm \cdot|\Lambda|$ & brf/rr $\pm r / \Lambda r$ & $c \Delta r / q \pm r / 1 \Delta$ & $\mathrm{d} F N / I T \pm 1 / 9 \Lambda$ \\
\hline \multicolumn{4}{|c|}{ PSA-NCAM هِسينإِى سولئوس } \\
\hline$a f \cdot / r \pm r / 4 r$ & $b_{1} \cdot / 4 \varepsilon \pm 1 / \cdot 0$ & $c r \cdot \mid \Lambda \pm r / \cdot \varphi$ & $\mathrm{d} r / r_{G} \pm r / \Delta q$ \\
\hline
\end{tabular}

${ }^{19}$ Shapiro wilks

${ }^{20}$ Leven

${ }^{21}$ One-way ANOVA

${ }^{22}$ Dunnett
${ }^{23}$ Treadmill with EAE

${ }^{24}$ Swim with EAE

${ }^{25}$ Morphology

${ }^{26}$ Neurodegenerative 


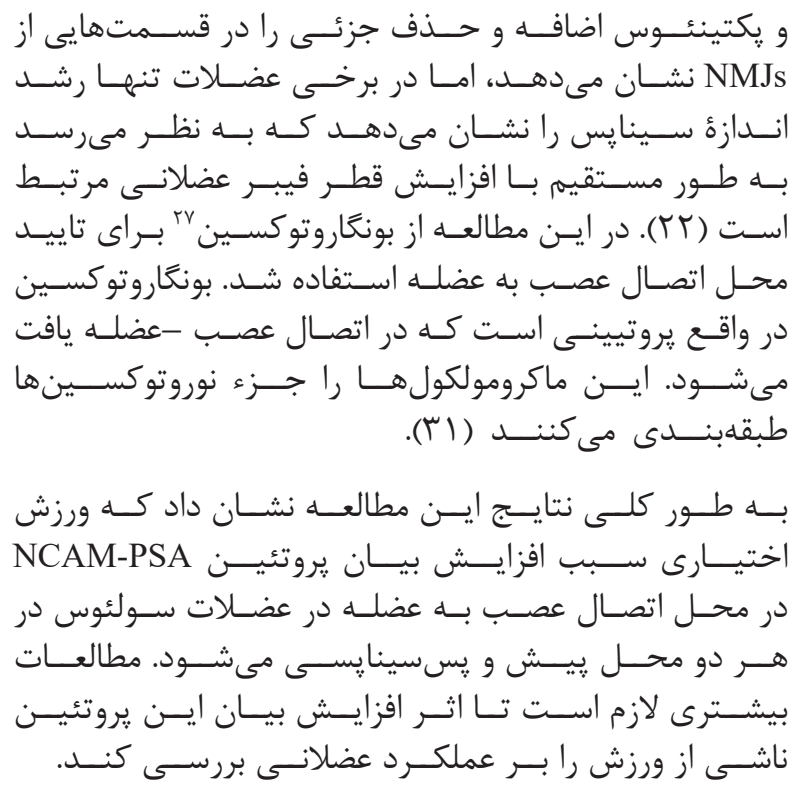

1. Hlavac RJ, Klaus R, Betts K, Smith SM, Stabio ME. Novel dissection of the central nervous system to bridge gross anatomy and neuroscience for an integrated medical curriculum. Anat Sci Educ. 2018; 11(2): 185-95.

2. Wang L, Wang FS, Gershwin ME. Human autoimmune diseases: a comprehensive update. J Intern Med. 2015; 278(4): 369-95.

3. Baecher-Allan C, Kaskow BJ, Weiner HL. Multiple sclerosis: mechanisms and immunotherapy. Neuron. 2018; 97(4): 742-68

4. Kamińska J, Koper OM, Piechal K, Kemona H. Multiple sclerosis - etiology and diagnostic potential. Postepy Hig Med Dosw. 2017; 71(0): 551-63.

5. Katz Sand I. Classification, diagnosis, and differential diagnosis of multiple sclerosis. Curr Opin Neurol. 2015; 28(3): 193-205.

6. Dendrou CA, Fugger L, Friese MA. Immunopathology of multiple sclerosis. Nature Reviews Immunology. 2015; 15(9): 545-58.

7. Raper J, Mason C. Cellular strategies of axonal pathfinding. Cold Spring Harbor perspectives in biology. 2010; 2(9): a001933.

8. El Maarouf A, Rutishauser U. Use of NCAM-PSAin repair of the central nervous system. Adv Exp Med Biol. 2010; 663: 137-47.

9. Maarouf AE, Yaw DM-L, Rutishauser U. Improved

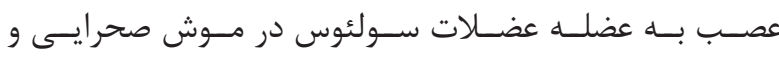

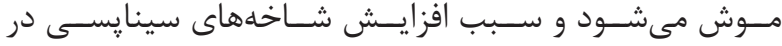

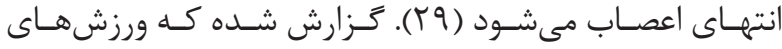

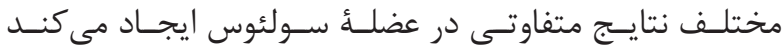

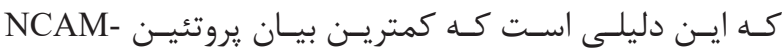

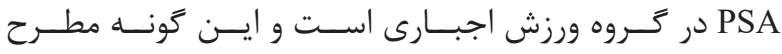

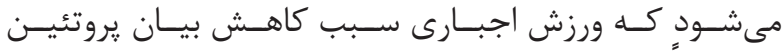

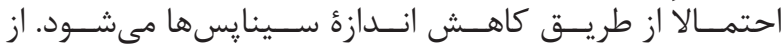

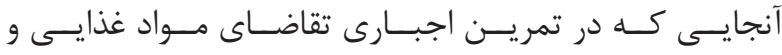

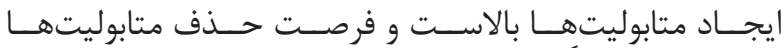

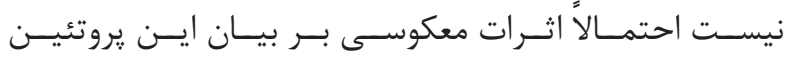

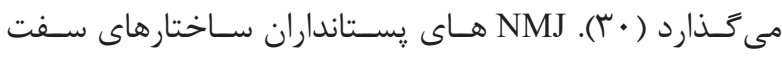

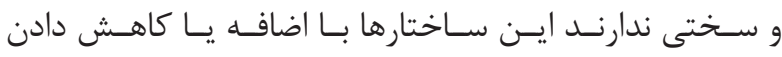

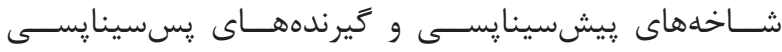

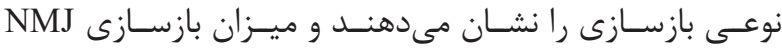

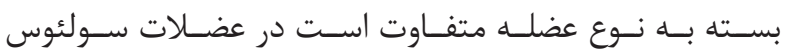

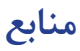

stem cell-derived motoneuron survival, migration, sprouting, and innervation with enhanced expression of polysialic acid. Cell Transplantation. 2015; 24(5): 797809.

10. Franz CK, Rutishauser U, Rafuse VF. Intrinsic neuronal properties control selective targeting of regenerating motoneurons. Brain. 2008; 131(6): 1492505 .

11. Caldow MK, Thomas EE, Dale MJ, Tomkinson GR, Buckley JD, Cameron-Smith D. Early myogenic responses to acute exercise before and after resistance training in young men. Physiol Rep. 2015; 3(9): e12511.

12. Reynolds ER, Ashbaugh AD, Hockenberry BJ, McGrew CA. multiple sclerosis and exercise: a literature review. Curr Sports Med Rep. 2018; 17(1): 31-5.

13. Cruickshank T, Reyes A, Ziman M. A systematic review and meta-analysis of strength training in individuals with multiple sclerosis or Parkinson's disease. Medicine (Baltimore). 2015; 94(4): doi: 10.1097/MD.

14. Souza-Teixeira F, Costilla S, Ayán C, García-López D, González-Gallego J, de Paz JA. Effects of resistance training in multiple sclerosis. Int J Sports Med. 2009; 30(4): 245-50.

15. Deschenes MR, Kressin KA, Garratt RN, Leathrum CM, Shaffrey EC. Effects of exercise training on neuromuscular junction morphology and pre- to post-synaptic coupling in young and aged rats. Neuroscience. 2016; 316: 167-77

${ }^{27}$ Bungarotoxin 
16. Neamţu MC, Rusu L, Neamţu OM, Enescu Bieru D, Marin MI, Croitoru IC, et al. Analysis of neuromuscular parameters in patients with multiple sclerosis and gait disorders. Rom J Morphol Embryol. 2014; 55(4): 1423-8.

17. Deschenes MR, Kressin KA, Garratt RN, Leathrum CM, Shaffrey EC. Effects of exercise training on neuromuscular junction morphology and pre- to post-synaptic coupling in young and aged rats. Neuroscience. 2016; 316: 167-77.

18. Talebi F, Ghorbani S, Chan WF, Boghozian R, Masoumi F, Ghasemi S, et al. MicroRNA-142 regulates inflammation and $\mathrm{T}$ cell differentiation in an animal model of multiple sclerosis. J Neuroinflammation. 2017; 14(1): 55. doi: 10.1186/s12974-017-0832-7.

19. Deschenes MR, Maresh CM, Crivello JF, Armstrong LE, Kraemer WJ, Covault J. The effects of exercise training of different intensities on neuromuscular junction morphology. J Neurocytol. 1993; 22(8): 603-15.

20. Ziliotto N, Zivadinov R, Jakimovski D, Baroni M, Tisato V, Secchiero P, et al. Plasma levels of soluble NCAM in multiple sclerosis. J Neurol Sci. 2019; 396: 36-41.

21. Werneburg S, Fuchs HLS, Albers I, Burkhardt H, Gudi V, Skripuletz T, et al. Polysialylation at early stages of oligodendrocyte differentiation promotes myelin repair. J Neurosci. 2017; 37(34): 8131-41.

22. Handschin C, Spiegelman BM. The role of exercise and PGC1alpha in inflammation and chronic disease. Nature. 2008; 454(7203): 463-46.

23. Nishimune H, Stanford JA, Mori Y. Role of exercise in maintaining the integrity of the neuromuscular junction. Muscle Nerve. 2014; 49(3) :315-24.
24. Li Y, Thompson WJ. Nerve terminal growth remodels neuromuscular synapses in mice following regeneration of the postsynaptic muscle fiber. Neurosci. 2011; 31(37): 13191-203.

25. Dalgas U, Stenager E. Exercise and disease progression in multiple sclerosis: can exercise slow down the progression of multiple sclerosis? Ther Adv Neurol Disord. 2012; 5(2): 81-95.

26. Cotman CW, Berchtold NC, Christie L-A. Exercise builds brain health: key roles of growth factor cascades and inflammation. Trends in Neurosciences. 2007; 30(9): 464-72.

27. Siette J, Westbrook RF, Cotman C, Sidhu K, Zhu W, Sachdev P, et al. Age-specific effects of voluntary exercise on memory and the older brain. Biol Psychiatry. 2013; 73(5): 435-42.

28. Battistuzzo CR, Callister RJ, Callister R, Galea MP. A systematic review of exercise training to promote locomotor recovery in animal models of spinal cord injury. J Neurotrauma. 2012; 29(8): 1600-13.

29. Deschenes MR, Roby MA, Glass EK. Aging influences adaptations of the neuromuscular junction to endurance training. Neuroscience. 2011; 190: 56-66.

30. Deschenes MR, Judelson DA, Kraemer WJ, Meskaitis VJ, Volek JS, Nindl BC, et al. Effects of resistance training on neuromuscular junction morphology. Muscle Nerve. 2000; 23(10): 1576-81.

31. Rajendran BK, Xavier Suresh M, Bhaskaran SP, Harshitha Y, Gaur U, Kwok HF. Pharmacoinformatic approach to explore the antidote potential of phytochemicals on bungarotoxin from indian krait, bungaruscaeruleus. Comput Struct Biotechnol J. 2018; 16: $450-61$ 